PROCEEDINGS OF THE

AMERICAN MATHEMATICAL SOCIETY

Volume 138, Number 1, January 2010, Pages 1-7

S 0002-9939(09)10086-2

Article electronically published on September 4, 2009

\title{
A NOTE ON CERTAIN KRONECKER COEFFICIENTS
}

\author{
L. MANIVEL
}

(Communicated by Jonathan I. Hall)

\begin{abstract}
We prove an explicit formula for the tensor square of an irreducible complex representation of the symmetric group defined by a rectangle of height two. We also describe part of the decomposition for the tensor product of representations defined by rectangles of heights two and four. Our results are deduced, through Schur-Weyl duality, from the observation that certain actions on triple tensor products of vector spaces are multiplicity free.
\end{abstract}

\section{INTRODUCTION}

Irreducible complex representations of the symmetric group $\mathcal{S}_{n}$ are well known to be indexed by partitions of $n$ in a natural way (see e.g. [Mc, I.7). We will denote by $[\lambda]$ the representation associated to the partition $\lambda$. A major unsolved problem is to find a general rule for the tensor product $[\lambda] \otimes[\mu]$ of two such representations. Equivalently, one would like a general rule for the computation of the Kronecker coefficients, which are defined as the multiplicities appearing in the formula

$$
[\lambda] \otimes[\mu]=\bigoplus_{\nu} k_{\lambda \mu \nu}[\nu] .
$$

In the setting of algebraic complexity theory, a specific instance of this general problem has been brought to the fore: can one compute the tensor square of an irreducible complex representation of the symmetric group defined by a rectangle partition? (See BLMW] for an overview.) If the rectangle has height one this is pretty obvious, since the corresponding representation is the trivial one. In this note we give an answer for the next case, that of a rectangle of height two. (Note that a rectangle of width two would lead exactly to the same answer, since one can pass from a partition to the dual one, in terms of representations of the symmetric group, simply by the product with the sign representation.)

We denote by $\ell(\lambda)$ the length of a partition $\lambda$, that is, the number of nonzero parts. A partition is said to be even (resp. odd) when all its nonzero parts are even (resp. odd).

Received by the editors October 8, 2008

2000 Mathematics Subject Classification. Primary 20C30, 15A72, 11S90.

(C)2009 American Mathematical Society Reverts to public domain 28 years from publication 
Our main result is the following:

Theorem 1. For any integer $n$, the tensor product $[n, n] \otimes[n, n]$ is a multiplicity free representation of $\mathcal{S}_{2 n}$. Its decomposition into irreducibles is

$$
[n, n] \otimes[n, n]=\bigoplus_{\substack{\lambda \text { even, }|\lambda|=2 n \\
\ell(\lambda) \leq 4}}[\lambda] \oplus \bigoplus_{\begin{array}{c}
\mu \text { odd, }|\mu|=2 n \\
\ell(\mu)=4
\end{array}}[\mu] .
$$

An independent and very different proof of this result can also be found in GWXZ]. It would be interesting to understand the splitting of $[n, n] \otimes[n, n]$ into its symmetric and skew-symmetric parts.

To state our second result, we introduce the following notation:

$$
[\lambda] \otimes_{\ell}[\mu]=\bigoplus_{\ell(\nu) \leq \ell} k_{\lambda \mu \nu}[\nu]
$$

Theorem 2. For any integer $n$, the partial tensor product $[2 n, 2 n] \otimes_{3}[n, n, n, n]$ is a multiplicity free representation of $\mathcal{S}_{4 n}$. Its decomposition into irreducibles is

$$
[2 n, 2 n] \otimes_{3}[n, n, n, n]=\underset{\substack{|\lambda|=2 n \\ \lambda_{2}+\lambda_{3}-\lambda_{1} \geq 0 \text { and even }}}{\bigoplus}[2 \lambda]
$$

\section{Schur-Weyl DuAlity AND multiplicity free aCtions}

In order to prove the previous two theorems we will restate them in terms of representations of general linear groups in a quite standard way. Recall the statement of the Schur-Weyl duality between representations of symmetric groups and of general linear groups (see e.g. [Ho] ): let $V$ be any finite-dimensional complex vector space, and $n$ any integer. Then the $\mathcal{S}_{n} \times G L(V)$-module $V^{\otimes n}$ decomposes as

$$
V^{\otimes n}=\bigoplus_{|\lambda|=n}[\lambda] \otimes S_{\lambda} V
$$

where $S_{\lambda} V$ denotes the Schur module of weight $\lambda$, which is an irreducible polynomial representation of $G L(V)$. For three vector spaces $U, V, W$, a straightforward consequence of taking $\mathcal{S}_{n}$-invariants in $(U \otimes V \otimes W)^{\otimes n}$ is that for three partitions $\lambda, \mu, \nu$ of $n$, the multiplicity of $S_{\lambda} U \otimes S_{\mu} V \otimes S_{\nu} W$ inside $\operatorname{Sym}^{n}(U \otimes V \otimes W)$ is equal to the Kronecker coefficient $k_{\lambda \mu \nu}$. In particular, if $U$ and $V$ have respective dimensions $d u$ and $d v$, with $(u, v)=1$, we deduce that

$$
\operatorname{Sym}(U \otimes V \otimes W)^{S L(U) \times S L(V)}=\bigoplus_{n \geq 0} \bigoplus_{|\lambda|=n u v} k_{(n v)^{u},(n u)^{v}, \lambda} S_{\lambda} W .
$$

Here $(n v)^{u}$ denotes the rectangular partition with $u$ parts all equal to $n v$, so that the corresponding Schur module of $U$ consists of $S L(U)$-invariants. This shows that Kronecker coefficients involving rectangular partitions are closely related to invariant theory. Indeed our two theorems above will be translated into the statements that two invariant algebras $\operatorname{Sym}(U \otimes V \otimes W)^{S L(U) \times S L(V) \times N}$ are polynomial algebras, where $N$ is a group of strictly upper triangular matrices in $S L(W)$.

Note that the complete invariant algebra $\operatorname{Sym}(U \otimes V \otimes W)^{S L(U) \times S L(V) \times S L(W)}$ is then also a polynomial algebra. This happens when the dimensions of the three spaces are either $(n, 2,2),(2,3,3),(2,3,4)$ or $(2,3,5)$. In the terminology of [Ka], 
these cases correspond to $\theta$-groups defined by the triple nodes of the Dynkin diagrams of type $D_{n+2}, E_{6}, E_{7}$ and $E_{8}$. The two cases we examine in this note are thus related to $D_{6}$ and $E_{7}$, respectively.

As is well known, multiplicity free actions of reductive groups can be detected by the existence of an open orbit for a Borel subgroup. We use this principle in the following setting: let $G$ and $H$ be two reductive groups with finite-dimensional representations $V$ and $W$. Let $B$ denote a Borel subgroup of $H$ and $N$ its unipotent radical. Suppose that $G \times B$ acts on $V \otimes W$ with an open orbit $\mathcal{O}$. Let $X_{1}, \ldots, X_{r}$ denote the boundary components of $\mathcal{O}$, that is, the irreducible hypersurfaces in its complement. Applying $[\mathrm{Br}]$, Proposition 3 of Chapter 3, we are led to the following conclusions:

- $X_{1}, \ldots, X_{r}$ have equations $f_{1}, \ldots, f_{r}$ which are semi-invariants of $B$ with linearly independent weights $\mu_{1}, \ldots, \mu_{r}$; in particular $r$ cannot exceed the rank of $H$.

- The algebra $\mathbb{C}[V \otimes W]^{G \times N}$ is a polynomial algebra over $f_{1}, \ldots, f_{r}$.

That $f_{1}, \ldots, f_{r}$ are semi-invariants of $B$ of weights $\mu_{1}, \ldots, \mu_{r}$ means that $f_{i}(b x)=$ $\mu_{i}(b) f_{i}(x)$ for all $x \in V \otimes W$ and $b \in B$. Moreover, as an $H$-module, the algebra of $G$-invariant functions on $V \otimes W$ is multiplicity free:

$$
\mathbb{C}[V \otimes W]^{G}=\bigoplus_{\mu \in \mathbb{Z}_{+} \mu_{1}+\cdots+\mathbb{Z}_{+} \mu_{r}} W_{\mu}
$$

if $W_{\mu}$ denotes the irreducible $H$-module of highest weight $\mu$. Indeed, such a component of $\mathbb{C}[V \otimes W]^{G}$ can be detected by its one-dimensional subspace of $N$-invariants.

In order to prove our two theorems, we will therefore just need to prove that the corresponding actions have open orbits and to identify the boundary components.

\section{Proof of Theorem 1}

Let $U$ and $V$ be two-dimensional vector spaces.

Lemma 1. Consider the action of $S L(U) \times S L(V)$ on the flag variety $\mathcal{F}(U \otimes V)$. The generic isotropy group of this action is a product of $\mathbb{Z}_{2}$ by the 8-element quaternion group.

Proof. Consider a general flag $W_{1} \subset W_{2} \subset W_{3} \subset U \otimes V$. The projective line $\mathbb{P} W_{2} \subset \mathbb{P}(U \otimes V)$ meets the quadric $Q=\mathbb{P} U \times \mathbb{P} V$ in two general points, which means that $W_{2}$ has a basis of the form $u_{0} \otimes v_{0}, u_{1} \otimes v_{1}$, where $u_{0}, u_{1}$ is a basis of $U$ and $v_{0}, v_{1}$ is a basis of $V$. Multiplying, if necessary, one of these vectors by a scalar, we may suppose that $W_{1}$ is the line in $W_{2}$ generated by $u_{0} \otimes v_{0}+u_{1} \otimes v_{1}$. Finally, $W_{3}$ is the kernel of a general linear form $\phi$ vanishing on $W_{2}$. Since in terms of the dual basis, $W_{2}^{\perp}=\left\langle u_{0}^{\vee} \otimes v_{1}^{\vee}, u_{1}^{\vee} \otimes v_{0}^{\vee}\right\rangle$, we can suppose that $\phi=u_{0}^{\vee} \otimes v_{1}^{\vee}-u_{1}^{\vee} \otimes v_{0}^{\vee}$. This means that $W_{3}$ is generated by $W_{2}$ and $u_{0} \otimes v_{1}+u_{1} \otimes v_{0}$.

Now it is straightforward to compute the stabilizer of our flag explicitly and to identify it with the product of $\mathbb{Z}_{2}$ by a copy of the 8-element quaternion group.

In fact, the only important thing to us is that this stabilizer is finite because of the following corollary. Let $W$ be a four-dimensional vector space and $B$ a Borel subgroup in $G L(W)$.

Corollary 1. The group $S L(U) \times S L(V) \times B$ has an open orbit in $U \otimes V \otimes W$. 
Proof. Consider a tensor $T \in U \otimes V \otimes W$ as a morphism $\phi_{T}: W^{\vee} \rightarrow U \otimes V$. For a generic $T$ this morphism is injective and maps the flag defining $B$ (or rather the orthogonal flag) to a generic flag in $U \otimes V$. By Lemma 1, $S L(U) \times S L(V)$ has an open orbit in the flag variety $\mathcal{F}(U \otimes V)$. Also, once the image flag is fixed, it is clear that $B$ acts transitively on the set of compatible injections.

As we explained above, the next step is to describe the boundary components of the open orbit. Let us denote by $F=\left(W_{1} \subset W_{2} \subset W_{3} \subset W\right)$ the flag whose stabilizer is the Borel subgroup $B$ of $G L(W)$, and by $F^{\perp}$ the orthogonal flag in $W^{\vee}$.

As in the proof of Lemma 1, we denote by $\phi_{T}: W^{\vee} \rightarrow U \otimes V$ the morphism defined by the tensor $T \in U \otimes V \otimes W$. We can describe the boundary components of the open orbit in $U \otimes V \otimes W$ by the following codimension one conditions:

(1) $\phi_{T}$ is not an isomorphism. The corresponding boundary component $X_{1}$ is the complement of the $S L(U) \times S L(V) \times G L(W)$-orbit. It is just the quartic hypersurface of equation $f_{1}=\operatorname{det} \phi_{T}$. This equation is a weight vector in $S_{22} U^{\vee} \otimes S_{22} V^{\vee} \otimes \bigwedge^{4} W^{\vee}=\bigwedge^{4}(U \otimes V)^{\vee} \otimes \bigwedge^{4} W^{\vee} \subset S^{4}(U \otimes V \otimes W)^{\vee}$. This means that the weight $\mu_{1}$ of $f_{1}$ is, written as a sequence of three partitions, $\mu_{1}=(22,22,1111)$.

(2) $\phi_{T}\left(\mathbb{P} W_{3}^{\perp}\right)$ belongs to the quadric $Q$. The corresponding boundary component $X_{2}$ is defined by the condition that $q\left(\phi_{T}\left(w^{\vee}\right)\right)=0$ if $w^{\vee}$ generates $W_{3}^{\perp}$ and $q$ denotes an equation of $Q$. Thus an equation $f_{2}$ of $X_{2}$ is a highest weight vector in $\bigwedge^{2} U^{\vee} \otimes \bigwedge^{2} V^{\vee} \otimes S_{2} W^{\vee}$. It has degree two and weight $\mu_{2}=(11,11,2)$.

(3) $\phi_{T}\left(\mathbb{P} W_{2}^{\perp}\right)$ is a tangent line to $Q$. This is the case if and only if $\phi_{T}\left(W_{2}^{\perp}\right)$ is generated by vectors of the form $u_{0} \otimes v_{0}$ and $u_{0} \otimes v_{1}+u_{1} \otimes v_{0}$. Considered as a line $\ell$ in $\Lambda^{2} W^{\vee}$, this means that $W_{2}^{\perp}$ is mapped by $\phi_{T}$ to the line generated by $u_{0}^{2} \otimes\left(v_{0} \wedge v_{1}\right) \oplus\left(u_{0} \wedge u_{1}\right) \otimes v_{0}^{2}$ in $\bigwedge^{2}(U \otimes V)=S^{2} U \otimes \bigwedge^{2} V \oplus$ $\bigwedge^{2} U \otimes S^{2} V$. Since the map $S^{2}\left(S^{2} U\right) \rightarrow S_{22} U$ kills any tensor of the form $\left(u^{2}\right)^{2}$, we deduce that $\phi_{T}$ maps $\ell^{2}$ to zero in $S_{22} U \otimes S_{22} V$. This implies that an equation $f_{3}$ of the corresponding boundary component $X_{3}$ is a highest weight vector in $S_{22} U^{\vee} \otimes S_{22} V^{\vee} \otimes S_{22} W^{\vee}$. It has degree four and its weight is $\mu_{3}=(22,22,22)$.

(4) $\phi\left(\mathbb{P} W_{1}^{\perp}\right)$ is a tangent plane to $Q$. This is similar to the case of $X_{2}$, up to duality. A hyperplane $H$ in $U \otimes V$ defines a line in $\bigwedge^{3}(U \otimes V)=$ $U \otimes V \otimes\left(\bigwedge^{2} U \otimes \bigwedge^{2} V\right)$, hence a line $\ell$ in $U \otimes V$ (the orthogonal line with respect to the polarity defined by $Q$ ). This hyperplane is tangent to the quadric $Q$ if and only if $\ell$ is contained in $Q$. This means that $X_{4}$ is defined by the condition that the composition

$$
S^{2}\left(\bigwedge^{3} W^{\vee}\right) \rightarrow S^{2}\left(\bigwedge^{3}(U \otimes V)\right)=S^{2}(U \otimes V) \otimes\left(\bigwedge^{2} U \otimes \bigwedge^{2} V\right)^{2} \rightarrow\left(\bigwedge^{2} U \otimes \bigwedge^{2} V\right)^{3}
$$

vanishes. Hence an equation $f_{4}$ of $X_{4}$ is a highest weight vector in $S_{33} U^{\vee} \otimes$ $S_{33} V^{\vee} \otimes S_{222} W^{\vee}$. It has degree six and weight $\mu_{4}=(33,33,222)$.

The four weights of $f_{1}, f_{2}, f_{3}, f_{4}$ are linearly independent. Since the rank of $G L(W)$ is four, we must have found all the boundary components, and we can conclude that

$$
\mathbb{C}[U \otimes V \otimes W]^{S L(U) \times S L(V) \times N}=\mathbb{C}\left[f_{1}, f_{2}, f_{3}, f_{4}\right],
$$


where $N$ denotes the unipotent radical of $B$. This implies that $\mathbb{C}[U \otimes V \otimes W]$ contains a copy of $S_{n, n} U^{\vee} \otimes S_{n, n} V^{\vee} \otimes S_{\lambda} W^{\vee}$ if and only if $\lambda$ is a nonnegative linear combination of the components of $\mu_{1}, \mu_{2}, \mu_{3}$ and $\mu_{4}$ on $W$, that is, the weights (1111), (2), (22) and (222). Moreover, in that case the multiplicity is equal to one.

Rephrasing this result via Schur-Weyl duality we get the statement of Theorem 1.

\section{Proof of Theorem 2}

For the proof of Lemma 2 we need to briefly recall the principle of castling transforms, introduced by Sato and Kimura SK. Consider a $G$-module $M$ of dimension $m$ and a vector space $N$ of dimension $n$. Suppose that $m>n$. A tensor $T$ in $M \otimes N$ can be identified to a linear map $\phi_{T}: N^{\vee} \rightarrow M$. If $\phi_{T}$ is injective, in particular for a generic $T$, the stabilizer of $T$ in $G \times G L(N)$ is canonically isomorphic to the stabilizer in $G$ of the image of $\phi_{T}$, considered as a point of the Grassmannian $G(n, M)$. But this Grassmannian is isomorphic with $G\left(n-m, M^{\vee}\right)$, and the generic stabilizer of the action of $G \times G L(N)$ on $M \otimes N$ is therefore isomorphic with the generic stabilizer of the action of $G \times G L(P)$ on $M^{\vee} \otimes P$, for $P$ a vector space of dimension $m-n$. Replacing $M \otimes N$ by $M^{\vee} \otimes P$ is precisely what Sato and Kimura call a castling transform. In the case where $M \otimes N$ is prehomogeneous and $m<2 n$, $M^{\vee} \otimes P$ is also prehomogeneous but of smaller dimension.

Let $U, V, W$ be complex vector spaces of respective dimension two, four and three. Let $B$ be a Borel subgroup of $G L(W)$.

Lemma 2. The group $S L(U) \times S L(V) \times B$ has an open orbit in $U \otimes V \otimes W$.

Proof. We claim that the generic isotropy group of the action of $S L(U) \times S L(V) \times$ $G L(W)$ on $U \otimes V \otimes W$ is a copy of $S L_{2}$, up to a finite group. To check this, we observe that $U \otimes V \otimes W$ is, according to the terminology of Sato and Kimura, a nonreduced prehomogeneous vector space, which means that it is related to smaller prehomogeneous spaces of the same type by certain castling transforms.

In order to apply this process to the case we are interested in, we first observe that the generic stabilizers of $S L(U) \times S L(V) \times G L(W)$ and $S L(U) \times G L(V) \times S L(W)$ on $U \otimes V \otimes W$ are equal, up to a finite group. Applying a castling transform with $G=S L(U) \times S L(W)$ acting on $U \otimes W$, we deduce that this generic stabilizer is the same as the generic stabilizer of the action of $S L(U) \times G L(Q) \times S L(W)$ on $U^{\vee} \otimes Q \otimes W^{\vee}$, where $Q$ has dimension $2 \times 3-4=2$. Up to a finite group, this is also the generic stabilizer of the action of $S L(U) \times S L(Q) \times G L(W)$, and after a new castling transform, we deduce that this is also the generic stabilizer of the action of $S L(U) \times S L(Q) \times G L(R)$ on $U \otimes Q^{\vee} \otimes R$, where now $R$ has dimension $2 \times 2-3=1$. Let us identify $U$ and $Q$, which are both two-dimensional. Then it is easy to see that the identity map $I \in U \otimes Q^{\vee}$ has generic stabilizer, so that this generic stabilizer is just a copy of $S L_{2}$ embedded diagonally in $S L(U) \times S L(Q)$.

We can keep track of this generic stabilizer along our two castling transforms. We start from the point in $U \otimes U^{\vee}=\operatorname{End}(U)$ defined by $I$. The corresponding point in $U^{\vee} \otimes U \otimes W^{\vee}$, where $W$ is identified with the orthogonal to $I$ in $\operatorname{End}(U)$ (the hyperplane $\operatorname{End}_{0}(U)$ of traceless matrices), is just the graph of the embedding of $\operatorname{End}_{0}(U)$ in $\operatorname{End}(U)$. Its isotropy is the image of $S L(U)$ in $S L\left(U^{\vee}\right) \otimes S L(U) \otimes$ $S L\left(W^{\vee}\right)$ given by the natural action of $S L(U)$ on each of the three spaces. Now we make our second castling transform to get a point in $U \otimes V \otimes W$, where $V$ is now identified with the kernel of the natural evaluation map $\operatorname{End}_{0}(U) \otimes U \rightarrow U$. The 
corresponding stabilizer is again a copy of $S L(U)$ embedded in $S L(U) \otimes S L(V) \otimes$ $S L(W)$ through its natural action on $U, V$ and $W$.

We can now check our claim: it can be translated into the assertion that the image of $S L(U)$ into $G L(W)$, where $W=\operatorname{End}_{0}(U)$, does not intersect a general Borel subgroup. But this is straightforward: such a Borel subgroup is defined by a line, generated by a generic traceless matrix $m$, and a hyperplane containing it, which can be defined as the orthogonal to a generic traceless matrix $n$ orthogonal to $m$. For an element of $S L_{2}$, preserving $m$ and $n$ forces it to belong to the intersection of two tori, and this intersection is finite.

Now we identify in $U \otimes V \otimes W$ the boundary components of the open orbit of $S L(U) \times S L(V) \times B$. They can be described in terms of the following codimension one conditions on a tensor $T \in U \otimes V \otimes W$, which will be best expressed in terms of certain auxiliary morphisms.

(1) First recall that $S L(U) \times S L(V) \times G L(W)$ has itself an open orbit whose complement is an irreducible hypersurface $X_{1}$ of degree 12 [Ka]. An equation $f_{1}$ of this hypersurface can be obtained as follows. Consider the morphism $\psi^{T}: U^{\vee} \otimes W^{\vee} \rightarrow V$ induced by $T$. Taking its second wedge power, we get an induced map

$$
\Psi^{T}: \bigwedge^{2} U^{\vee} \otimes S^{2} W^{\vee} \hookrightarrow \bigwedge^{2}\left(U^{\vee} \otimes W^{\vee}\right) \stackrel{\wedge^{2} \psi^{V}}{\longrightarrow} \bigwedge^{2} V
$$

between two vector spaces of the same dimension, six. We can thus let $f_{1}=\operatorname{det} \Psi^{T}$, an invariant of degree 12 and weight $\mu_{1}=(66,3333,444)$.

(2) Restricting $\psi^{T}$, we can define a morphism

$$
\psi_{1}^{T}: U \otimes W_{1}^{\perp} \rightarrow V
$$

between two vector spaces of the same dimension, four. We can thus define another boundary component $X_{2}$ by the condition that this is not an isomorphism. An equation of this hypersurface is $f_{2}=\operatorname{det} \psi_{1}^{T}$, a semiinvariant of degree 4 and weight $\mu_{2}=(22,1111,22)$.

(3) To describe our next boundary component, we need to recall that there exists an invariant nondegenerate skew-symmetric form of $S^{3} U$, or equivalently an equivariant morphism

$$
\omega: \bigwedge^{2}\left(S^{3} U\right) \rightarrow\left(\bigwedge^{2} U\right)^{3}
$$

Now consider the morphism

$$
\phi_{T}: \bigwedge^{3} W^{\vee} \longrightarrow S^{3} U \otimes \bigwedge^{3} V \simeq S^{3} U \otimes \bigwedge^{4} V \otimes V^{\vee}
$$

induced by $T$. (Note that $\bigwedge^{3} W$ and $\wedge^{4} V$ are both one dimensional.) Taking its square, we get a map

$$
\Phi_{T}: S^{2}\left(\bigwedge^{3} W^{\vee}\right) \rightarrow \bigwedge^{2}\left(S^{3} U\right) \otimes\left(\bigwedge^{4} V\right)^{2} \otimes \bigwedge^{2} V^{\vee} \rightarrow\left(\bigwedge^{2} U\right)^{3} \otimes\left(\bigwedge^{4} V\right)^{2} \otimes \bigwedge^{2} V^{\vee}
$$

where the last arrow is induced by $\omega$. The image of $\Phi_{T}$ defines, up to scalar, a skew-symmetric form $\omega_{T}$ on $V$. On the other hand, we can restrict the morphism $\Psi^{T}$ to the line $\bigwedge^{2} U^{\vee} \otimes S^{2} W_{2}^{\perp} \subset \bigwedge^{2} U^{\vee} \otimes S^{2} W^{\vee}$. 
Its image is, up to scalar, an element of $\Omega_{T}$ of $\bigwedge^{2} V$. We can thus define a boundary component $X_{3}$ by the condition that the natural pairing $\left\langle\omega_{T}, \Omega_{T}\right\rangle=0$. An equation $f_{3}$ of this hypersurface has degree 8 and weight $\mu_{3}=(44,2222,422)$.

Since we have found three boundary components and $\operatorname{dim}(U \otimes V \otimes W)-\operatorname{dim}(S L(U) \times$ $S L(V) \times B)=3$, we must have found all the boundary components and we can conclude that

$$
\mathbb{C}[U \otimes V \otimes W]^{S L(U) \times S L(V) \times B}=\mathbb{C}\left[f_{1}, f_{2}, f_{3}\right] .
$$

The weights of $f_{1}, f_{2}, f_{3}$ are independent, as expected, and we deduce that $\mathbb{C}[U \otimes V \otimes W]$ contains a component $S_{n, n} U \otimes S_{n, n} V \otimes S_{\lambda} W$ if and only if $\lambda=$ $\left(\lambda_{1}, \lambda_{2}, \lambda_{3}\right)$ is a nonnegative linear combination of the components of $\mu_{1}, \mu_{2}$ and $\mu_{3}$ on $W$, that is, (422), (444) and (422). Because of the identity

$$
\lambda=\frac{\lambda_{1}-\lambda_{2}}{2}(422)+\frac{\lambda_{3}+\lambda_{2}-\lambda_{1}}{4}(444)+\frac{\lambda_{2}-\lambda_{3}}{2}(22),
$$

this is equivalent to the conditions that $\lambda$ be even and that $\lambda_{3}-\lambda_{1}-\lambda_{2}$ be a nonnegative multiple of four. Moreover, in that case the multiplicity is equal to one. Rephrasing this result via Schur-Weyl duality we get the statement of Theorem 2.

\section{REFERENCES}

[GWXZ] Garsia, A., Wallach, N., Xin, G., Zabrocki, M., Kronecker coefficients via symmetric functions and constant term identities, preprint, 2008.

[Br] Brion, M., Invariants et covariants des groupes algébriques réductifs, in Théorie des invariants et Géométrie des variétés quotients, Travaux en cours 61, Hermann, 2000.

[BLMW] Buergisser, P., Landsberg, J.M., Manivel, L., Weyman, J., An overview of mathematical issues arising in the geometric complexity theory approach to VP vs. VNP, arXiv:0907.2850.

[Ho] Howe, R., Perspectives on invariant theory: Schur duality, multiplicity-free actions and beyond, in The Schur lectures (1992) (Tel Aviv), 1-182, Israel Math. Conf. Proc. 8, Bar-Ilan Univ., Ramat Gan, 1995. MR1321638 (96e:13006)

[Ka] Kac, V.G., Some remarks on nilpotent orbits, J. Algebra 64 (1980), no. 1, 190-213. MR575790 (81i:17005)

[Mc] Macdonald, I.G., Symmetric functions and Hall polynomials, second edition, Oxford University Press, 1995. MR 1354144 (96h:05207)

[SK] Sato, M., Kimura, T., A classification of irreducible prehomogeneous vector spaces and their relative invariants, Nagoya Math. J. 65 (1977), 1-155. MR0430336 (55:3341)

Institut Fourier, University of Grenoble I, 100 Rue des Mathematiques, 38402 St. Martin d'Heres, France 